\title{
Introduction to the Special Issue on Caucasian Languages
}

\section{Ioana Chitoran and Denis Creissels}

The six articles collected in this special issue are a selection of talks given at the "Workshop for young linguists - Caucasian languages" organized by the editors in Lyons, France, April 8-9, 2009. The workshop was supported by funding from the "Collegium de Lyon" and the Laboratoire "Dynamique du Langage" - CNRS, Université Lyon 2.

Our main goal in organizing the workshop and in editing the current issue is to bring together young linguists working on the relatively less studied Caucasian languages, and to offer them a forum for the exchange of ideas, for comparison of data and of different methods of analysis, as well as for potential future collaborations. Unlike many other linguistic areas or language families, the linguistic study of the Caucasus seems to proceed with interruptions, alternating between periods of higher and lower activity, determined by a complex interaction of factors. To name just a few: the unsettled political situation in the area, the variable depth of linguistic tradition and formal study of language in different countries of the Caucasus, the status of a given language as a majority or minority language, the presence or absence of orthography, of literacy, of written vs. oral cultural tradition. A gap is noticeable even in the current issue. Of the three existing Caucasian language families, the Northeast (Nakh-Daghestanian) and the South (Kartvelian) families are well represented, with three articles each, but the Northwest family happens to be missing.

We hope that the current issue will contribute to increase interest in the study of Caucasian languages, whose linguistic systems present many aspects that are typologically rare and theoretically challenging. Most experts currently agree (Tuite 2007) that the three Caucasian language families are not genetically related, and thus are also interesting from the perspective of areal linguistics. As Comrie (2008) points out, few features characterize the Caucasus as a single linguistic area.

Caucasian languages do not currently have a dedicated conference series, or a journal that meets the international standards of serving as a forum for the exchange of ideas among linguists working on this linguistic area, or on a Caucasian language family. Therefore, the current issue is also meant to remind the linguistic community of this existing gap, and to help increase the visibility of Caucasian languages in linguistic research, both for the purposes of language documentation and for testing hypotheses about theoretical models.

The following six articles are presented in this volume, listed here in alphabetical order of the authors:

- Diana Forker "Finiteness in Hinuq"

- Zaira Khalilova "Evidentiality in Tsezic Languages"

- Silvia Kutscher "On the Expression of Spatial Relations in Ardeşen-Laz"

- René Lacroix "Ditransitive Constructions in Laz"

- Zarina Molochieva "Aspect in Chechen"

- Manana Topadze-Gäumann “The Expression of Evidentiality in Georgian” 
The articles deal with topics of syntax and semantics that are currently at the forefront of research in linguistic typology: TAM and evidentiality, clause-combining, ditransitive constructions, expression of spatial relations. In keeping with the editorial statement of Linguistic Discovery, all the articles presented here are based on the description and analysis of primary linguistic data.

\section{References}

Comrie, Bernard. 2008. Linguistic diversity in the Caucasus. Annual Review of Anthropology 37. 131-143

Tuite, Kevin. 2007. The rise and fall and revival of the Ibero-Caucasian hypothesis. Historiographia Linguistica 35 \#1. 23-82 